\title{
Probing the magnetic ground state of the molecular dysprosium triangle with muon spin relaxation
}

\author{
Z. Salman,,${ }^{1,2, *}$ S. R. Giblin, ${ }^{3}$ Y. Lan, ${ }^{4}$ A. K. Powell,${ }^{4}$ R. Scheuermann, ${ }^{2}$ R. Tingle,,${ }^{3}$ and R. Sessoli ${ }^{5}$ \\ ${ }^{1}$ Clarendon Laboratory, Department of Physics, Oxford University, Parks Road, Oxford OX1 3PU, United Kingdom \\ ${ }^{2}$ Laboratory for Muon Spin Spectroscopy, Paul Scherrer Institut, CH-5232 Villigen PSI, Switzerland \\ ${ }^{3}$ ISIS Facility, Rutherford Appleton Laboratory, Chilton, Oxfordshire OX11 OQX, United Kingdom \\ ${ }^{4}$ Institut für Anorganische Chemie, Karlsruher Institut für Technologie (KIT), Engesserstr. 15, 76131 Karlsruhe, Germany \\ ${ }^{5}$ Dipartimento di Chimica, Universitá di Firenze \& INSTM, via della Lastruccia 3, 50019 Sesto Fiorentino, Italy
}

(Received 3 November 2010; published 22 November 2010)

\begin{abstract}
We present zero field muon spin lattice relaxation measurements of a Dysprosium triangle molecular magnet. The local magnetic fields sensed by the implanted muons indicate the coexistence of static and dynamic internal magnetic fields below $T^{*} \sim 35 \mathrm{~K}$. Bulk magnetization and heat capacity measurements show no indication of magnetic ordering below this temperature. We attribute the static fields to the slow relaxation of the magnetization in the ground state of $\mathrm{Dy}_{3}$. The fluctuation time of the dynamic part of the field is estimated to be $\sim 0.55 \mu$ s at low temperatures.
\end{abstract}

DOI: $10.1103 /$ PhysRevB.82.174427

\section{INTRODUCTION}

Recent development in the field of molecular magnetism has produced idealized model systems that allow the investigation of quantum phenomena in nanomagnets. ${ }^{1}$ Examples include slow relaxation in high spin molecules, ${ }^{2}$ quantum tunneling of the magnetization, ${ }^{3,4}$ topological quantum phase interference, ${ }^{5,6}$ and quantum coherence. ${ }^{7-9}$ Within these systems, antiferromagnetic clusters, rings or triangles,, 910 have been considered for possible use in quantum computation, due to longer decoherence time as a result of weaker intercluster dipolar interactions. ${ }^{8}$ Here we investigate a recently synthesized trinuclear cluster $\left[\mathrm{Dy}_{3}\left(\mu_{3}\right.\right.$ $\left.-\mathrm{OH})_{2} \mathrm{~L}_{3} \mathrm{Cl}\left(\mathrm{H}_{2} \mathrm{O}\right)_{5}\right] \mathrm{Cl}_{3}$ (where $\mathrm{L}$ is the anion of orthovanillin), ${ }^{11}$ hereafter abbreviated as $\mathrm{Dy}_{3}$ (see Fig. 1). This compound exhibits two important properties; a system with a nonmagnetic ground state as well as slow relaxation of the magnetization which is typical of some high spin clusters. ${ }^{12}$ Magnetization measurements have been explained using a model of three $\mathrm{Dy}^{3+}$ moments arranged on a triangle, each with a large Ising anisotropy axis in the plane rotated by $120^{\circ}$ relative to its neighbors (see Fig. 1). A small antiferromagnetic interaction $(\sim 0.2 \mathrm{~K})$ between $\mathrm{Dy}^{3+}$ results in a nonmagnetic, $S=0$, ground state characterized by a vortex spin-chirality.

In this paper we present a zero field (ZF) muon spin relaxation ( $\mu \mathrm{SR})$ study of $\mathrm{Dy}_{3}$. In this technique polarized muons are used as local spin probes of the microscopic magnetic structure of the individual $\mathrm{Dy}_{3}$ molecules. We find that below $T^{*} \sim 35 \mathrm{~K}$ the local magnetic field experienced by the muons has two components, dynamic and static. The static component is direct evidence of the slow relaxation of the magnetization in the ground state of $\mathrm{Dy}_{3}$, while the dynamic component is used to measure the fluctuations time of the $\mathrm{Dy}_{3}$ ground spin state directly. This is possible due to the unique local perspective of the muon.

\section{EXPERIMENTAL}

The $\mu$ SR experiments were performed on the DOLLY spectrometer at the Paul Scherrer Institute, Switzerland. In
PACS number(s): 75.75. $-\mathrm{c}, 75.45 .+\mathrm{j}, 75.50 . \mathrm{Xx}, 76.75 .+\mathrm{i}$

these experiments $100 \%$ polarized (along the beam direction, $z$ ) positive muons are implanted in the sample. Each implanted muon decays (lifetime $\tau_{\mu}=2.2 \mu \mathrm{s}$ ) emitting a positron preferentially in the direction of its polarization at the time of decay. Using appropriately positioned detectors, one measures the asymmetry of the muon beta decay along $z$ as a function of time $A(t)$, which is proportional to the time evolution of the muon spin polarization. $A(t)$ depends on the distribution of internal magnetic fields and their temporal fluctuations. Further details on the $\mu$ SR technique may be found in Ref. 13.

The composition and structure of $\mathrm{Dy}_{3}$ sample was confirmed using X-ray diffraction and magnetization measurements. The powder sample was placed in a ${ }^{4} \mathrm{He}$ gas flow cryostat to measure the muon spin relaxation in the temperature range between 1.6 and $300 \mathrm{~K}$. Heat capacity measurements at ZF and in the temperature range between 2 and 100 $\mathrm{K}$ were performed on the same sample using a quantum design physical properties measurement system. Additionally, magnetization measurements in the same temperature range were performed using a quantum design magnetic properties measurement system.

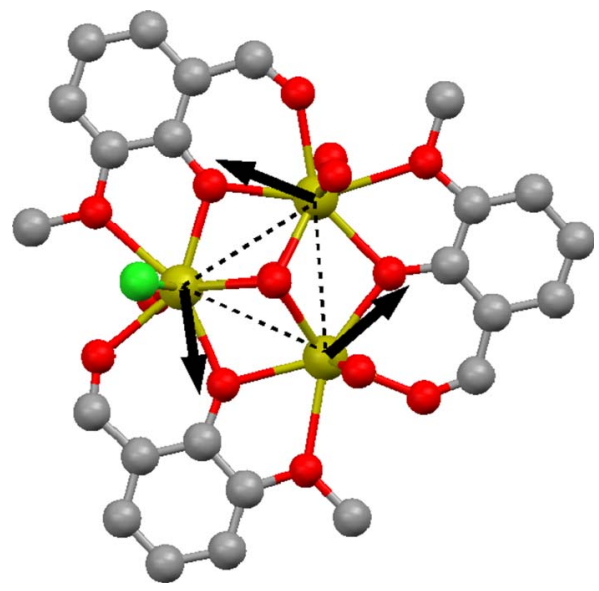

FIG. 1. (Color online) The magnetic core of the $\mathrm{Dy}_{3}$ molecules. The yellow, red, green and gray balls represent the $\mathrm{Dy}, \mathrm{O}, \mathrm{Cl}$, and $\mathrm{C}$ atoms, respectively. 


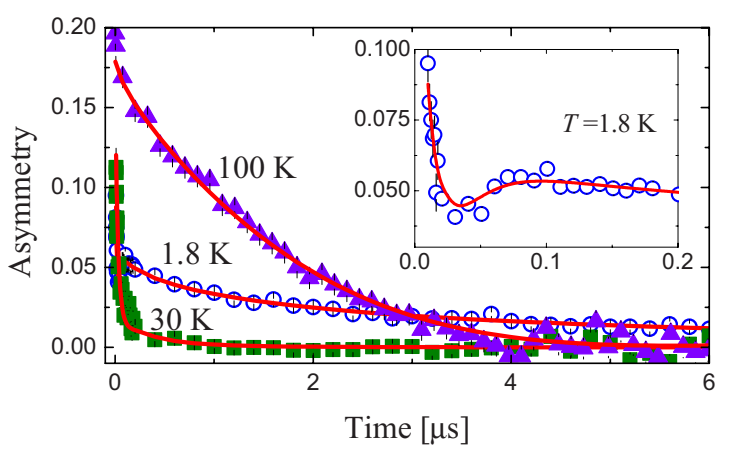

FIG. 2. (Color online) The asymmetry as a function of time at three different temperatures. The inset shows the asymmetry at very short times, where the dip is clear evidence for a static local field experienced by the muons. The solid lines are fits to Eq. (2).

\section{RESULTS}

Example muon spin relaxation curves measured in $\mathrm{ZF}$ are presented in Fig. 2. Note that at low temperatures, the asymmetry exhibits a dip at early times (inset of Fig. 2), followed by a recovery and then relaxation at longer times. In contrast, at high temperature the asymmetry relaxes almost exponentially from its initial value to zero. The low temperature relaxation curves are evidence that the internal magnetic field experienced by the implanted muons in $\mathrm{Dy}_{3}$ contains two contributions; a static (time independent) component and a fluctuating component. As we discuss below, the static component is direct evidence of the slow relaxation of the individual $\mathrm{Dy}_{3}$ (or $\mathrm{Dy}^{3+}$ ) magnetic moments. Generally, when muons experience a distribution of static magnetic fields $\rho\left(\mathbf{B}-\mathbf{B}_{s}, \Delta\right)$, where $\mathbf{B}_{s}$ is the average static field and $\Delta$ is the root mean square of the field distribution, then the asymmetry follows a static Kubo-Toyabe function:

$$
\begin{gathered}
A_{\mathrm{KT}}(t)=A_{0} \int \rho\left(\mathbf{B}-\mathbf{B}_{s}, \Delta\right) G_{z}(t) d^{3} B, \\
G_{z}(t)=\cos ^{2} \theta+\sin ^{2} \theta \cos (\gamma B t) .
\end{gathered}
$$

Here $\gamma=135.5 \mathrm{MHz} / \mathrm{T}$ is the muon gyromagnetic ratio, $\theta$ is the angle between the initial muon spin and the local static magnetic field $\mathbf{B}$ which is averaged over a powder sample. For example, if $\mathbf{B}_{s}=0$ then the asymmetry is at its maximum value at $t=0$, it exhibits a dip at $t \sim 1 / \Delta$ and recovers to $\sim 1 / 3$ its initial value at long times. Depending on the form of $\rho$, e.g., Gaussian or Lorentzian, the relaxation follows a Gaussian Kubo-Toyabe (GKT), $A_{\mathrm{GKT}}$, or a Lorentzian KuboToyabe (LKT), $A_{\mathrm{LKT}}$, respectively. However, if in addition to the static field component a small fluctuating field $B_{d}(t)$ is present, then the $1 / 3$ tail continues to relax to zero. ${ }^{14-16}$ In this case the relaxation can be described by a phenomenological function: a LKT or GKT multiplied by a suitable dynamic relaxation function. ${ }^{17}$

In our case, the asymmetry measured in $\mathrm{Dy}_{3}$ at all temperatures was found to fit best to LKT multiplied by a square root exponential relaxation,

$$
A(t)=A_{\mathrm{LKT}}(t) e^{-\sqrt{\lambda t}} .
$$

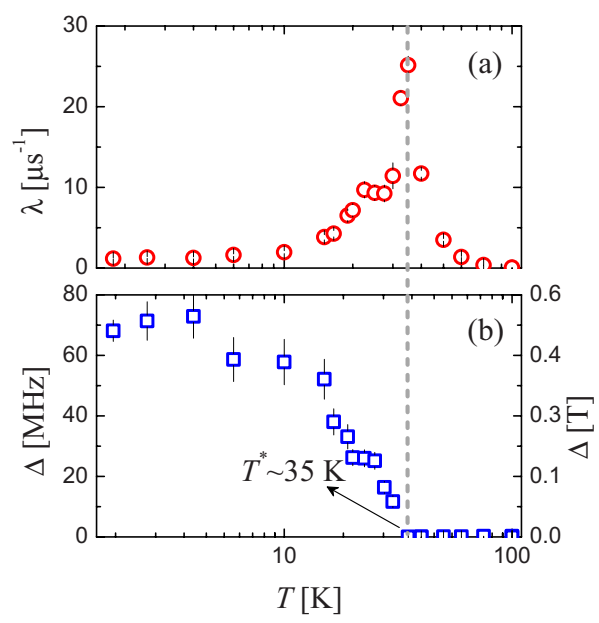

FIG. 3. (Color online) The values of (a) $\lambda$ and (b) $\Delta$ obtained from fits of the relaxation curves as a function of temperature.

where $\lambda$ is the relaxation rate, containing information regarding the dynamics of the local field. In particular, at low temperatures where $\Delta>\lambda, A(t)$ is almost identical to the well known dynamic LKT function and hence $\lambda=2 / 3 \tau,{ }^{14,15}$ where $\tau$ is the fluctuation time of the local magnetic field. ${ }^{18}$ The square root exponential relaxation reflects the averaging of the relaxation behavior of muons stopping in multiple inequivalent sites. ${ }^{15,19-22}$

The parameters $\lambda$ and $\Delta$ obtained from the fits are shown in Figs. 3(a) and 3(b), respectively. Note that at high temperatures $\lambda$ is small and $\Delta \approx 0$. As the temperature is decreased $\lambda$ increases sharply while $\Delta$ remains zero. At $T^{*}$ $\sim 35 \mathrm{~K}, \lambda$ peaks and $\Delta$ becomes nonzero. Finally, at lower temperatures $\lambda$ decreases again while $\Delta$ saturates at $\sim 70 \mathrm{MHz}$, corresponding to a width in field of $\sim 0.45 \mathrm{~T}$ [right side axis of Fig. 3(b)]. These temperature dependencies are commonly seen in materials undergoing magnetic ordering at $T_{c}=T^{*}{ }^{23}$ In such cases the increase of $\lambda$ above $T_{c}$ is attributed to a critical slowing down of the fluctuations and $\Delta$ is proportional to the order parameter (local magnetization). ${ }^{23,24}$ This behavior is quite unusual, and exhibits a sharp transition from a paramagnetic to an ordered/ static regime, or the sudden appearance of static magnetic fields in the system. Such static fields are rarely observed in SMMs studied by $\mu$ SR. ${ }^{22}$

Triggered by these findings we measured the magnetization and heat capacity to confirm that there is no evidence of long range magnetic ordering in the $\mathrm{Dy}_{3}$ system. As can be seen in Fig. 4, bulk magnetization measurements in $50 \mathrm{mT}$ show no indication of ordering at $\sim 35 \mathrm{~K} .{ }^{11,12}$ Similarly, heat capacity measurements in $\mathrm{ZF}$ show no anomalies around this temperature. Therefore, the observed static fields can be associated with either (I) an ordering only on the local scale, e.g., the freezing (or slowing down) of the fluctuations of the individual $\mathrm{Dy}^{3+}$ magnetic moments, or (II) a magnetic ground state where the $\mathrm{Dy}^{3+}$ moments order is such a way that they do not cancel each other, resulting in a ground state with a nonvanishing magnetic moment. The latter possibility is ruled out by bulk magnetization measurements which confirm a nonmagnetic ground state in $\mathrm{Dy}_{3}$ molecules. ${ }^{11,12}$ 


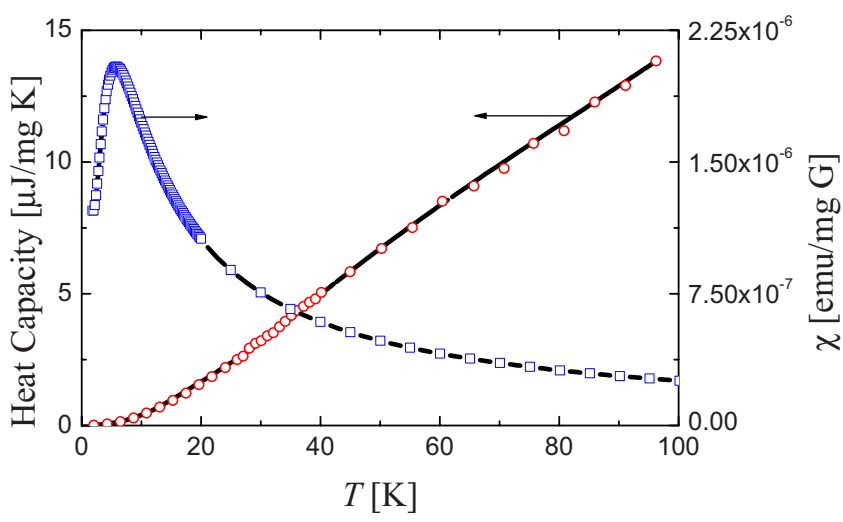

FIG. 4. (Color online) The heat capacity measured in ZF (circles, left axes) and susceptibility measured in $50 \mathrm{mT}$ (squares, right axes) of $\mathrm{Dy}_{3}$ as a function of temperature. There is no evidence for any anomaly at $35 \mathrm{~K}$. The solid lines are a guide for the eyes.

\section{DISCUSSION}

We start by discussing the local static field contribution and its origin. Assuming that the muons occupy sites which are relatively far from the $\mathrm{Dy}_{3}$ magnetic core (i.e., at a distance much larger than the distance between different Dy ions in the molecule), one expects that the static dipolar field experienced by the muons will decrease and vanish as the magnetic moment of $\mathrm{Dy}_{3}$ decreases at low temperature. However, since muons sense a strong nonvanishing static field at low temperatures (up to $\Delta_{0} \sim 0.45 \mathrm{~T}$ ), we conclude that the muons occupy sites which are close to the magnetic core, and therefore are sensitive to the microscopic magnetic structure of the molecules. One such possibility is a site near the $\mathrm{Cl}$ ion shown in Fig. 1. This site is negatively charged and therefore may attract the positively charged implanted muons. On this site, the dipolar fields from the three individual Dy ions do not cancel each other even if the $\mathrm{Dy}^{3+}$ moments are arranged in a nonmagnetic ground state configuration. Instead, the $\mathrm{Dy}^{3+}$ closest to the $\mathrm{Cl}$ contributes mostly to the magnetic field experienced by the muon (roughly 20 times larger). For example, assuming $\Delta_{0}$ is due to a dipolar field of a single $\mathrm{Dy}^{3+}$ moment we expect that the distance between this moment and its neighboring muon is roughly $\sim 5 \AA$. This distance is similar to that between the $\mathrm{Cl}$ mentioned above and its neighboring $\operatorname{Dy}^{3+}(\sim 2.7 \AA)$. Therefore, we conclude that this static field is direct evidence of the slow relaxation of individual $\mathrm{Dy}_{3}$ moments, which can be associated with single $\mathrm{Dy}^{3+}$ ion anisotropy. ${ }^{12}$

These measurements exhibits the power of a local probe measurement and its advantages over conventional bulk magnetization measurements in resolving the magnetic properties of individual magnetic molecules, and the microscopic magnetic structure in general. Moreover, the observed local static magnetic fields, despite the nonmagnetic ground state, reflect the peculiar nature of the spin vortex arrangement and the Ising/classical type interactions between the spins which give a nonmagnetic doublet, i.e., two distinct magnetic states. Although muons cannot detect the sense of chirality of the ground state, they provide evidence of its static magnetic nature.

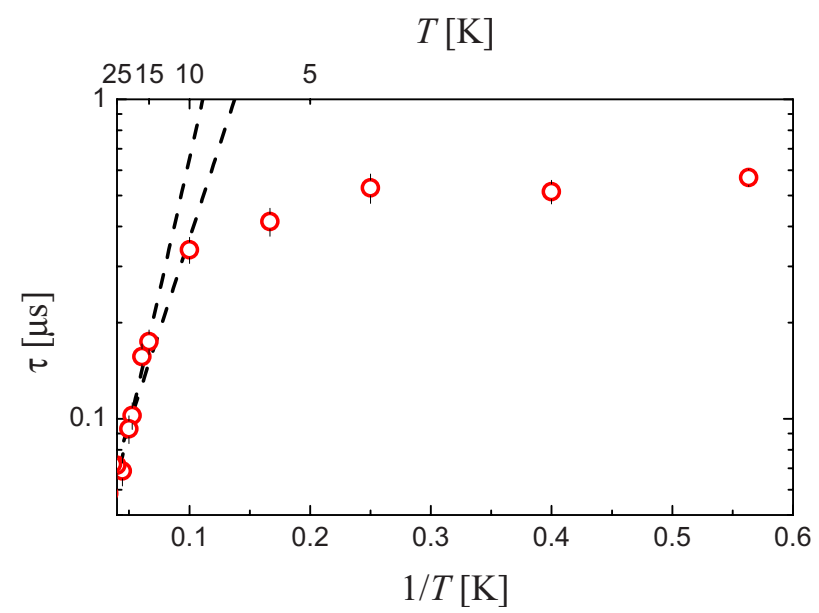

FIG. 5. (Color online) The fluctuation time of the local field experienced by muons as a function of temperature. The dashed lines indicate the range of parameters for fits to Arrhenius law.

Next, we investigate the dynamic properties of the local field experienced by the implanted muons. As we mentioned earlier, the low temperature relaxation $\lambda$ can be used to extract the fluctuation time $\tau$ of the local field. In Fig. 5 we plot $\tau$ as a function of temperatures (below $\sim 25 \mathrm{~K}$ where $\Delta$ $>\lambda$ ). We find that $\tau \sim 0.1 \mu \mathrm{s}$ at $\sim 20 \mathrm{~K}$, increases gradually as the temperature is decreased and saturates at $\sim 0.55 \mu \mathrm{s}$ below $\sim 4 \mathrm{~K}$. These fluctuations are due to fluctuations of the $\mathrm{Dy}^{3+}$ magnetic moments near the stopping site of the muon. In a very simplified picture, muons are sensitive to $\tau$ only when it is shorter than the muon's lifetime $\left(\tau_{\mu}\right.$ $=2.2 \mu \mathrm{s}$ ). When $\tau \gtrsim \tau_{\mu}$ we expect the muon to experience a static field component. This is consistent with the extracted values of $\tau$; in the range of a fraction of a $\mu \mathrm{s}$. In contrast to this scale of dynamics, ac susceptibility measurements of the relaxation time of the magnetization at zero applied field have shown that it follows an Arrhenius law ${ }^{12}$

$$
\tau_{m}=\tau_{m}^{0} \exp \left(U_{m} / T\right),
$$

where $\tau_{m}^{0}=2.5(5) \times 10^{-7} \mathrm{~s}$ and $U_{m}=36(2) \mathrm{K}$. The similarity between the temperature dependence of our results and those extracted from ac susceptibility is striking. However, the low temperature saturation value of the fluctuation time is an order of magnitude longer in the ac susceptibility measurements. For comparison, fits of $\tau$ in the range $10-25 \mathrm{~K}$ to Eq. (3) yield an activation barrier $U=27-39 \mathrm{~K}$ and attempt time $\tau^{0}=(1.4-2.5) \times 10^{-8} \mathrm{~s}$ (dashed lines in Fig. 5). This discrepancy indicates that although both $\mu \mathrm{SR}$ and ac susceptibility measurements confirm the slow relaxation of the magnetization, the techniques do not measure the same scale of dynamics. However, given that these techniques and their temporal sensitivities are different, there are two possible explanations of the discrepancy. One possibility is that while the dynamics measured with ac susceptibility are those of collective bulk magnetization, $\mu$ SR measures fluctuations of individual molecules or $\mathrm{Dy}^{3+}$ moments within it. For example, studies in $\mathrm{Ni}_{10}$ magnetic molecules have shown that while ac susceptibility exhibits slow relaxation of bulk magnetization, nuclear magnetic resonance (NMR) measurements confirm the pres- 
ence of fast single molecule dynamics. ${ }^{25,26}$ This was interpreted as slowing down of the bulk magnetization due to a resonant phonon trapping mechanism which prevents thermalization of the bulk magnetization but allows fast spin flipping of the individual molecular moments. ${ }^{26}$ Another possibility is that each techniques is sensitive to a different dynamic process, which may not be even related. The dynamics of the bulk magnetization was associated to fluctuations of the $\mathrm{Dy}_{3}$ magnetization in the plane of the $\mathrm{Dy}^{3+}$ triangle. However, fluctuations out of the plane cannot be ruled out. This type of fluctuations may be faster, and therefore more effective in causing muon spin lattice relaxation. We believe that NMR measurements in $\mathrm{Dy}_{3}$ should be sensitive to both scales of dynamics, and therefore would provide valuable information that could clarify the discrepancy between bulk magnetization measurements and $\mu \mathrm{SR}$.

\section{CONCLUSIONS}

In conclusion, we find that muons implanted in $\mathrm{Dy}_{3}$ observe a fluctuating field at high temperatures. Below $\sim 35 \mathrm{~K}$ the local field has two components, static and dynamic. Bulk magnetization and heat capacity measurements rule out the existence of long range ordering associated with the observed temperature dependence of the local field. Instead, the observed static local magnetic field is direct evidence of the slow relaxation of the magnetization. Our measurements exhibit that in spite of the peculiar nature of the $\mathrm{Dy}^{3+}$ spins vortex arrangement which produces a nonmagnetic doublet, a local probe such as muons can detect their static magnetic nature. Therefore, although no net magnetic moment is present, they can be used, in principle, to store magnetic information (sense of chirality) with the advantage of long coherence time due to the small intermolecular dipolar couplings. Finally, we find that the time scale of low temperature dynamics detected with $\mu \mathrm{SR}$ is an order of magnitude shorter than that extracted from ac susceptibility measurements. The different time scale of dynamics could be due to the sensitivity of the different techniques to different relaxation processes or a difference between fluctuations of collective and individual moments.

\section{ACKNOWLEDGMENTS}

We would like to thank Andreas Suter for reading the paper and helpful discussions. This research project has been supported by the European Commission under the 7th Framework Programme through the 'Research Infrastructures' action of the 'Capacities' Programme, Contract No. CP-CSA_INFRA-2008-1.1.1 Number 226507-NMI3. The $\mu \mathrm{SR}$ measurements were performed at the Swiss Muon Source $(\mathrm{S} \mu \mathrm{S})$, at the Paul Scherrer Institute in Villigen, Switzerland.

*zaher.salman@psi.ch

${ }^{1}$ D. Gatteschi, R. Sessoli, and J. Villain, Molecular Nanomagnets (Oxford University Press, New York, 2006).

${ }^{2}$ R. Sessoli, D. Gatteschi, A. Caneschi, and M. Novak, Nature (London) 365, 141 (1993).

${ }^{3}$ J. R. Friedman, M. P. Sarachik, J. Tejada, and R. Ziolo, Phys. Rev. Lett. 76, 3830 (1996).

${ }^{4}$ L. Thomas, F. Lionti, R. Ballou, D. Gatteschi, R. Sessoli, and B. Barbara, Nature (London) 383, 145 (1996).

${ }^{5}$ W. Wernsdorfer and R. Sessoli, Science 284, 133 (1999).

${ }^{6}$ W. Wernsdorfer, R. Sessoli, A. Caneschi, D. Gatteschi, and A. Cornia, EPL 50, 552 (2000).

${ }^{7}$ E. del Barco, A. D. Kent, E. C. Yang, and D. N. Hendrickson, Phys. Rev. Lett. 93, 157202 (2004).

${ }^{8}$ A. Morello, P. C. E. Stamp, and I. S. Tupitsyn, Phys. Rev. Lett. 97, 207206 (2006)

${ }^{9}$ S. Bertaina, S. Gambarelli, T. Mitra, B. Tsukerblat, A. Müller, and B. Barbara, Nature (London) 453, 203 (2008).

${ }^{10}$ A. Ardavan, O. Rival, J. J. L. Morton, S. J. Blundell, A. M. Tyryshkin, G. A. Timco, and R. E. P. Winpenny, Phys. Rev. Lett. 98, 057201 (2007).

${ }^{11}$ J. Tang, I. Hewitt, N. T. Madhu, G. Chastanet, W. Wernsdorfer, C. E. Anson, C. Benelli, R. Sessoli, and A. K. Powell, Angew. Chem., Int. Ed. 45, 1729 (2006).

${ }^{12}$ J. Luzon, K. Bernot, I. J. Hewitt, C. E. Anson, A. K. Powell, and R. Sessoli, Phys. Rev. Lett. 100, 247205 (2008).

${ }^{13}$ S. L. Lee, S. H. Kilcoyne, and R. Cywinski, Muon Science (SUSSP and Institute of Physics Publishing, Bristol, 1998).

${ }^{14}$ R. S. Hayano, Y. J. Uemura, J. Imazato, N. Nishida, T. Yamazaki, and R. Kubo, Phys. Rev. B 20, 850 (1979).

${ }^{15}$ Y. J. Uemura, T. Yamazaki, D. R. Harshman, M. Senba, and E. J. Ansaldo, Phys. Rev. B 31, 546 (1985).

${ }^{16}$ Y. J. Uemura, in Muon Science: Muons in Physics, Chemistry and Materials, edited by S. Lee, S. Kilcoyne, and R. Cywinski (SUSSP and Institute of Physics Publishing, 1998), Chap. 4.

${ }^{17}$ Y. Fudamoto et al., Phys. Rev. B 65, 174428 (2002).

${ }^{18}$ Simulations show that the dynamic LKT and the phenomenological function $A_{\mathrm{LKT}}(t) e^{-\lambda t}$ are identical when $\Delta>\lambda$.

${ }^{19}$ A. Lascialfari, Z. H. Jang, F. Borsa, P. Carretta, and D. Gatteschi, Phys. Rev. Lett. 81, 3773 (1998).

${ }^{20}$ Z. Salman, A. Keren, P. Mendels, V. Marvaud, A. Scuiller, M. Verdaguer, J. S. Lord, and C. Baines, Phys. Rev. B 65, 132403 (2002).

${ }^{21}$ S. J. Blundell, F. L. Pratt, T. Lancaster, I. M. Marshall, C. A. Steer, S. L. Heath, J. F. Letard, T. Sugano, D. Mihailovic, and A. Omerzu, Polyhedron 22, 1973 (2003).

${ }^{22}$ F. Branzoli, M. Filibian, P. Carretta, S. Klyatskaya, and M. Ruben, Phys. Rev. B 79, 220404 (2009).

${ }^{23}$ G. M. Kalvius, D. R. Noakes, and O. Hartmann, in Handbook on the Physics and Chemistry of Rare Earths, edited by G. Lander, K. Gschneider, and L. Eyring (Elsevier, New York, 2001), Vol. 32, p. 55.

${ }^{24}$ Z. Salman, T. J. Parolin, K. H. Chow, T. A. Keeler, R. I. Miller, D. Wang, and W. A. MacFarlane, Phys. Rev. B 73, 174427 (2006).

${ }^{25}$ S. Carretta, P. Santini, G. Amoretti, M. Affronte, A. Candini, A. Ghirri, I. S. Tidmarsh, R. H. Laye, R. Shaw, and E. J. L. McInnes, Phys. Rev. Lett. 97, 207201 (2006).

${ }^{26}$ M. Belesi et al., Phys. Rev. Lett. 102, 177201 (2009). 